\title{
Preditores do uso do Facebook pelos adolescentes portugueses: Contributos de um estudo exploratório
}

\author{
Predictors of Facebook use among Portuguese \\ adolescents: Contributes of an exploratory study
}

\author{
Paulo César Dias ${ }^{1}$ \\ Universidade Católica Portuguesa, Portugal \\ José António García del Castillo ${ }^{2}$ \\ Álvaro García del Castillo-López ${ }^{3}$ \\ Universidad Miguel Hernández, Elche, España
}

\begin{abstract}
Resumo. A generalização do uso das redes sociais online, tem trazido oportunidades e constrangimentos ao mesmo tempo-ou até mais depressa-que a investigação e o conhecimento sobre este tópico. Neste trabalho, pretendemos explorar o papel de variáveis pessoais e psicossociais no uso do Facebook, recorrendo a um conjunto de questionários de autorrelato junto de uma amostra de 386 adolescentes portugueses. Os resultados obtidos permitem verificar diferenças na utilização em função do género, uma relação positiva com a idade, a autorregulação e autoestima, sendo a interação com pares da sua rede de contactos offline o mais forte preditor para o uso das redes sociais. Estes dados sugerem o papel desta aplicação como um meio para manter o contacto com os pares.
\end{abstract}

Palavras-chave. Uso do Facebook, adolescentes, autoestima, autorregulação, pares.

Abstract. The widespread use of social networks has brought opportunities and constraints at the same time, even faster than research and knowledge on this topic. In this paper, we intend to explore the role of personal and psychosocial variables in Facebook use, considering a set of self-report questionnaires to a sample of 386 Portuguese adolescents. We found gender differences in social network use, a positive relationship with age, self-regulation and self-esteem, with peer interaction with offline contacts as the strongest predictor for social network use. These data suggest the role of this application as a mean to maintain contact with peers.

Keywords. Facebook use, adolescents, self-esteem, self-regulation, peers.

\footnotetext{
${ }^{1}$ Paulo César Dias. Universidade Católica Portuguesa, Portugal. Dirección postal: Faculdade de Filosofia e Ciências Sociais, Praça da Faculdade, no 1, 4710-297 Braga. E-mail: pcdias@,braga.ucp.pt

2 José António García del Castillo. Universidad Miguel Hernández, Elche, España. E-mail: jagr@umh.es

${ }^{3}$ Álvaro García del Castillo-López. Universidad Miguel Hernández, Elche, España. E-mail: alvaro.garciac@umh.es
} 


\section{Introdução}

O estudo das redes sociais é, há décadas, um campo de estudo muito fértil, nas diferentes disciplinas. $\mathrm{Na}$ revisão da literatura, facilmente encontramos referências já no início do século XIX (Deans, 2008), especialmente associado à antropologia e à sociologia, no estudo do comportamento e nas relações em sociedades mais ou menos complexas ou estruturadas (Deans, 2008; Mitchell, 1974). Também na investigação psicológica, este tem sido um conceito muito estudado, especialmente no âmbito da psicologia social como no âmbito da saúde, seja pelo impacto das relações no conhecimento de si mesmo e do outro, seja pela forte correlação entre a rede social de apoio com a saúde e bem-estar.

O entusiasmo e pertinência deste campo de estudo podem ser verificados em diferentes áreas disciplinares, como são exemplo as edições especiais apresentadas recentemente pela Health Psychology (Martire \& Franks, 2014), Social Science \& Medicine (Tsai \& Papachristos, 2015), os artigos apresentados nas séries da Anual Reviews sobre este tópico (e.g. Campbell, 2013; Smith \& Christakis, 2008; Umberson, Crosnoe \& Reczek, 2010; Videras, 2013), ou os estudos de revisão e meta-análises neste âmbito (e.g. Ell, 1984; Faber \& Wasserman, 2002; Jeon \& Goodson, 2015; Rico-Uribe et al., 2016). Com o avanço tecnológico e a chamada revolução da web 1.0 para a 2.0 , novas ferramentas e possibilidades de interação mediada pela tecnologia surgiram. E diversos estudos se debruçam sobre a maneira como estas ferramentas estão a influenciar a forma como nos relacionamos com os outros e connosco mesmos (e.g. Boyd \& Ellison, 2007; Castells, 2009; Baym, 2010). Se, num primeiro momento, o aparecimento da Internet, e especificamente da World Wide Web, teve um impacto decisivo na forma como os sujeitos passaram a aceder e a obter informação (Carvalho, 2008), o início dos anos 2000 trouxe um novo salto. Em 2004, Tim O’Reilly e a MediaLive International sugerem o aparecimento da Web 2.0, em que a internet deixa de ser apenas um "repositório do conhecimento humano" (BernersLee, Cailliau, Loutonen, Nielsen, \& Secret, 1994), para permitir maior partilha, capacidade de edição e criação de conteúdos por cada utilizador. $\mathrm{O}$ aparecimento destas ferramentas permitiu múltiplas possibilidades de interação e colaboração dando um papel ativo ao utilizador, que deixa de ser apenas um recetor passivo para passar a ser um co construtor da rede (O’Reilly, 2006).

$\mathrm{Na}$ chamada revolução da Web 2.0, uma das ferramentas mais populares e das que mais entrou no quotidiano de todos foram as redes sociais online. Uma das redes sociais mais utilizada é o Facebook. Lançada em 2004, por Mark Zuckerberg e Eduard Saverin para o estabelecimento/fortalecimento de relações da Universidade de Harvard, acabou por se expandir para outras universidades, a estudantes do ensino secundário, empresas, etc. A generalização do seu uso tem trazido oportunidades e constrangimentos ao mesmo tempo-ou até mais depressa-que as linhas de investigação e o conhecimento sobre este tópico.

Estando a ganhar terreno globalmente, discute-se se estarão também a influenciar os nossos modelos de interação social. Embora muito se discuta sobre a utilização, especialmente junto dos mais jovens, são raros os estudos no nosso contexto sobre a utilização de redes sociais por adolescentes. Os primeiros estudos têm centrado na adaptação de instrumentos (Dias, Garcia del Castillo, Castillo-López \& Sorte, 2015; Pontes, Andreassen \& Griffiths, 2016; Pontes, Patrão \& Griffiths, 2014), embora apontem já diferenças de género, sendo superior a utilização mais intensiva pelos rapazes, e em função da idade, com maior utilização dos mais jovens. Dados que vão genericamente de acordo com a literatura internacional centrada neste tópico (e.g. Andreassen, Pallesen \& Griffiths, 2016; Kross et al., 2013; McAndrew \& Jeong, 2012).

Contudo, são menos os estudos que relacionam com outras variáveis potencialmente implicadas no seu uso ou no seu efeito, como o bem-estar, a autoestima, a autorregulação, o suporte social, ansiedade, depressão ou outras (e.g. Andreassen, Pallesen \& Griffiths, 2016; Blachio, Przepiorka \& Pantic, 2016; Kross et al., 2013). Nesse sentido, com o presente estudo pretendemos perceber em que medida o uso de Facebook pelos adolescentes se relaciona com variáveis pessoais e 
psicossociais, nomeadamente com a autoestima e autorregulação, no sentido de identificar preditores do uso destas ferramentas. Se a maioria dos estudos se centra em jovens adultos, especialmente pela maior facilidade de recolha de dados, importa perceber como estes comportamentos ocorrem em idades mais precoces para o poder compreender e intervir.

\section{Redes sociais online na adolescência}

Boyd e Ellison (2007), definem estas redes sociais online como serviços baseados na internet que permitem aos sujeitos construir um perfil (onde incluem os seus interesses, filiação, adicionam imagens, etc.) que é partilhado com outros utilizadores que pertencem ao mesmo sistema. Com base nesse perfil, é possível ao utilizador gerir e partilhar informação com outros utilizadores com quem partilham comunicação, ver e acompanhar as atividades das pessoas dessa lista de relações (Boyd \& Ellison, 2007). Estas redes sociais permitem manter e reforçar as relações sociais com pessoas que já se conhecem, as também designadas de relações offline (Boyd \& Ellison, 2007; Capua, 2012; Lampe, Ellison \& Steinfield, 2007), com quem os indivíduos já convivem ou partilham um interesse comum. Para além destas, permitem ainda o estabelecimento de novas relações entre pessoas que, de outra forma não se poderiam conhecer, as designadas por relações online (Boyd \& Ellison, 2007; Lampe, Ellison \& Steinfield, 2007; Grieve, Indian, Witteveen, Tolan \& Marrington, 2013).

Embora exista uma perceção de que estas ferramentas se dediquem essencialmente a fins recreativos, diversos tem sido os trabalhos que estudam e sugerem a sua importância na criação do sentido de comunidade, partilha de experiências e recursos entre profissionais (Hew, 2011; Uribe-Tirado \& EchavarríaRamírez, 2008) ou na divulgação de serviços e eventos, por exemplo, de bibliotecas académicas (Charnigo \& Barnett-Ellis, 2007; Hendrix, Chiarella, Hasman, Murphy \& Zafron, 2009). Também na área da saúde, Boulos e Weeler (2007) discutem como a Web 1.0, e posteriormente a Web 2.0, permitem aproximar profissionais de saúde e pacientes, promover a proximidade, um contacto mais próximo e mais dinâmico. De facto, parecem ter o potencial de aumentar o sentido de pertença numa comunidade, reduzir o isolamento social (Boulos \& Weeler, 2007), aparecendo positivamente associadas ao capital social, às relações com pares e auto-estima (Ellison, Steinfield \& Lampe, 2007; Johnston, Tanner, Lalla \& Kawalski, 2013; Kalpidou, Coustin \& Morris, 2011; Steinfield, Ellison \& Lampe, 2008). Os utilizadores acabam por se sentir socialmente mais envolvidos, sendo as redes sociais o meio para o reforço das relações, do feedback e das respostas dos amigos. E esses dados têm sido encontrados em estudos em que se recorrem amostras apenas com utilizadores de redes sociais (Steinfield, Ellison \& Lampe, 2008) como utilizadores/nãoutilizadores (Ellison, Steinfield \& Lampe, 2007), em áreas urbanas, mas especialmente em áreas mais rurais, com menor acesso a meios de comunicação e de formação especializada (Boulos \& Weeler, 2007; Buckley, Hastings \& Mottershead, 2009).

Acompanhando o entusiasmo gerado à volta da utilização das redes sociais têm também sido reforçadas preocupações com o seu uso. Especialmente junto dos mais novos, a muitas vezes apelidada "net generation" (Tapscott, 1998) ou dos nativos digitais (Cornu, 2011; Prensky, 2001), muito se discute sobre as implicações das tecnologias no seu desenvolvimento (Bennett, Maton \& Kervin, 2008). Estudos realizados em diversos países mostram que os adolescentes tendem a ser utilizadores mais intensivos do que os adultos (Andreassen, Pallesen \& Griffiths, 2016; Boyd, 2008; McAndrew \& Jeong, 2012; Valkenburg \& Peter, 2009), encontrando-se frequentemente uma diminuição do uso destas redes sociais com a idade em diversos países. No entanto, nem sempre os dados sejam totalmente claros na comparação com grupos de outras faixas etárias (Calvani, Fini, Ranieri \& Picci, 2012; Helsper \& Eynon, 2009).

$\mathrm{E}$ os seus principais preditores dependem muito das variáveis estudadas. De entre elas, as sociodemográficas têm sido recorrentemente identificadas. Assim, os utilizadores mais frequentes tendem a ser os rapazes (Drabowicz, 2014; Kuss \& Griffiths, 2012; Subrahmanyam, Reich, Waechter \& Espinoza. 2008), 
tende a diminuir com a idade (Junco, 2015; Pfeil, Arjan \& Zaphiris, 2009), e com menor desempenho escolar ou envolvimento na escola (Drabowicz, 2014; Junco, 2011, 2015). Também o número de amigos tende a ser considerado um dos mais fortes preditores (Khana, Wohn \& Ellison, 2014), que faz com que o uso apareça relacionado em alguns estudos com a autoestima (Gonzales \& Hancock, 2011; Vogel, Rose, Roberts \& Eckles, 2014; Wilcox \& Stephen, 2013). Existindo menos investigação sobre o papel da autorregulação no uso da internet, um preditor importante da resiliência e ajustamento dos sujeitos (Garcia del Castillo, Dias \& Perim, 2012; Gardner, Dishion \& Connell, 2008), a exploração destes processos pode dar-nos pistas para a compreensão destes comportamentos e dar pistas para a intervenção (Wilcox \& Stephen, 2013). Nesse contexto, o presente trabalho pretende explorar o uso do Facebook por adolescentes, perspetivando o papel de variáveis individuais e psicossociais, como a autoestima e autorregulação, neste comportamento.

\section{Método}

\section{Amostra}

A amostra foi constituída por 386 adolescentes portugueses, a maioria rapazes $(n=235,60.9 \%)$, com idades entre os 14 e os 18 anos $(M=16.50, D P$ $=1.03)$. Como se percebe da tabela abaixo, os pais dos estudantes da amostra tinham, na sua maioria, habilitações ao nível do $2^{\circ}$ ou $3^{\circ}$ ciclo do ensino básico. Esta foi uma amostra de conveniência, nomeadamente proximidade geográfica com o centro de investigação, recolhida na zona norte do país, num contexto de nível socioeconómico médio. Foram incluidos todos os alunos que apresentaram a autorização dos seus encarregados de educação.

\section{Instrumentos}

Questionário sociodemográfico. Foi utilizado um questionário, construído pelos autores, para recolher dados que permitam caracterizar a amostra, nomeadamente, idade, género, ano de escolaridade, escolaridade dos pais, mas também alguns dados sobre o uso do Facebook, nomeadamente, quantos amigos tem no Facebook, quantos dos amigos no Facebook conhece pessoalmente, quanto tempo por dia passa no Facebook.

Escala de Intensidade de utilização do Facebook (Ellison, Steinfield \& Lampe, 2007), um instrumento composto por 13 itens, respondidos de forma likert, desde a opção 1-discordo fortemente-até 5-concordo fortemente, permite avaliar a intensidade da utilização do Facebook, a sua utilização com os contactos offline e para o estabelecimento de contactos online. A primeira dimensão permite avaliar os hábitos de utilização do Facebook, considerando a frequência, duração mas também a intensidade e a importância desta rede social na vida do indivíduo. A segunda dimensão permite perceber a regularidade do uso do Facebook para estabelecer/manter contacto com pessoas da sua rede social quotidiana, como os familiares, colegas ou amigos. A última dimensão permite perceber em que medida os indivíduos utilizam o Facebook para procurar novas relações ou conhecer pessoas novas a partir da rede social. Este instrumento também tem sido utilizado junto da população portuguesa, tendo sido adaptado e com bons valores de validade e fidelidade, dado o valor de alfa de Cronbach de .94 (Dias, Garcia del Castillo, Castillo-López \& Sorte, 2015).

Escala de Autoestima Global de Rosenberg (1965), um instrumento constituído por 10 itens referentes ao modo como cada indivíduo se sente em relação a si próprio(a), que são respondidos numa escala de opções fechada desde 1 (concordo totalmente) a 6 (discordo totalmente). Esta escala tem sito objeto de vários estudos de adaptação para o contexto português, tendo sido também testada junto de adolescentes com resultados adequados, seja em termos de validade num modelo bi-direccional, com valores de fidelidade entre e .63 na atitude negativa em relação ao self e .74 na atitude positiva em relação ao self (Romano, Negreiros \& Martins, 2007).

Escala de Autorregulação de Schwarzer (1999), um instrumento com 10 itens, que permite avaliar uma perspetiva disposicional da autorregulação, isto é, a capacidade de controlo atenção do indivíduo em comportamentos dirigidos à realização de objetivos desejados. Os itens são respondidos numa escala de resposta fechada, desde Nada 
Tabela 1

Descrição da amostra

\begin{tabular}{lccc}
\hline Variável & Resposta & Frequência & Percentagem \\
\hline Género & Masculino & 235 & 60.9 \\
& Feminino & 151 & 39.1 \\
Escolaridade do Pai & $1^{\circ}$ Ciclo & 83 & \\
& $2^{\text {o }}$ Ciclo & 147 & 21.5 \\
& $3^{\circ}$ Ciclo & 77 & 38.1 \\
& Secundário & 69 & 19.9 \\
& Superior & 3 & 17.9 \\
Escolaridade da Mãe & $1^{\circ}$ Ciclo & 87 & 0.8 \\
& $2^{\text {o Ciclo }}$ & 135 & 22.5 \\
& $3^{\circ}$ Ciclo & 79 & 35.0 \\
& Secundário & 77 & 20.5 \\
& Superior & 3 & 19.9 \\
\hline
\end{tabular}

Verdadeiro (1) a Mesmo Verdadeiro (4). Foi já testada e adaptada junto de adolescentes portugueses, tendo sido obtidos bons indicadores de validade e fidelidade de .67 (Dias, Garcia del Castillo \& Schwarzer, 2008).

\section{Procedimentos}

Para a recolha de dados, os autores começaram pela autorização dos estabelecimentos de ensino, após apresentação dos objetivos e da metodologia de recolha de dados, seguido do consentimento aos pais / encarregados de educação. Os instrumentos foram aplicados em horário escolar, na sala de aula, em momentos combinados com os professores. Os dados foram recolhidos de forma totalmente anónima e a participação voluntária, podendo os alunos desistir em qualquer momento. A informação recolhida foi codificada e analisada com recurso ao programa de tratamento estatístico Statistical Package for Social Sciences, v. 15.

\section{Resultados}

Num primeiro momento, foram efetuados um conjunto de análises estatísticas para perceber eventuais diferenças na utilização do Facebook, no autoestima e autorregulação em função de variáveis pessoais. Como se percebe na tabela seguinte, os dados sugerem que a autoestima e autorregulação são aproximadamente iguais entre rapazes e raparigas, assim como a intensidade de utilização do Facebook. Contudo, os rapazes parecem apresentar uma maior utilização da rede social para manter os contactos offline $\left[t\left({ }_{375}\right)=2.898, p=.004\right]$ e para novos contactos ditos online $\left[t\left(_{375}\right)=4.854, p<.01\right]$. Os tamanhos de efeito, calculados com o d de Cohen (1992) podem ser considerados baixos $(d=.31)$ ou médio $(d=.52)$.

Já no que diz respeito à idade, verifica-se uma relação positiva com a utilização da rede social $(r=$ $.148, p<.01)$ e autoestima $(r=.140, p<.01)$, o que significa maior intensidade no uso desta ferramenta, assim como maior autoestima entre os adolescentes mais velhos. A escolaridade da mãe aparece associada a algumas das variáveis, nomeadamente do uso do Facebook $(r=-.103, p<.05)$ e autorregulação $(r=$ $-.113, p<.05)$, não sendo encontrada nenhuma relação com a escolaridade do pai $(\phi>.05)$. Estes dados sugerem que quanto maior a escolaridade da mãe, menor a intensidade na utilização da rede e menor a autorregulação dos adolescentes. 
Tabela 2

Uso do Facebook, autoestima e autorregulação em função do género.

\begin{tabular}{lcccccccc}
\hline & \multicolumn{2}{c}{ Masculino } & \multicolumn{2}{c}{ Feminino } & \multicolumn{2}{c}{ Inf. Estatística } & \multicolumn{2}{c}{ Efeito } \\
\hline & $M$ & $D P$ & $M$ & $D P$ & $t$ & $g l$ & $p$ & $d$ \\
\cline { 2 - 9 } Uso do Facebook & 2.968 & 1.072 & 2.830 & 1.079 & 1.213 & 375 & .226 & - \\
Com rede offline & 2.924 & 1.115 & 2.593 & 1.022 & 2.898 & 375 & .004 & .31 \\
Para contactos online & 3.121 & 1.424 & 2.414 & 1.294 & 4.854 & 375 & .000 & .52 \\
Autoestima & 29.820 & 4.870 & 30.331 & 4.584 & -.366 & 374 & .714 & - \\
Autorregulação & 27.680 & 4.544 & 27.848 & 4.006 & -1.010 & 371 & .313 & - \\
\hline
\end{tabular}

Tabela 3

Uso do Facebook, autoestima e autorregulação em função da idade e escolaridade dos pais.

\begin{tabular}{lccc}
\hline & Idade $(r)$ & $\begin{array}{c}\text { Escolaridade } \\
\text { do Pai }(r s)\end{array}$ & $\begin{array}{c}\text { Escolaridade } \\
\text { da Mãe }(r s)\end{array}$ \\
\hline Uso do Facebook & & $.148^{* *}$ & -.032 \\
Com rede offline & .057 & -.039 & $-.103^{*}$ \\
Para contactos online & .097 & -.004 & -.067 \\
Autoestima & $.140^{* *}$ & .037 & -.086 \\
Autorregulação & .084 & -.101 & $-.113^{*}$ \\
\hline
\end{tabular}

Nota. ** Correlação significativa para 0.01

De acordo com os dados, tentamos explorar a relação entre as variáveis e o desempenho escolar. Como se percebe na tabela seguinte, foi encontrada uma relação significativa entre a autoestima e as classificações no último período a Língua Portuguesa $(r=.178, p<.01)$ e Matemática $(r=.173, p<.01) \mathrm{e}$ entre o uso com contactos offline e as classificações a Língua Portuguesa $(r=-.127, p<.05)$.

Quando se tenta perceber a relação entre o uso do Facebook, autoestima e autorregulação, o número de amigos na rede e o tempo por dia, aproximadamente, que os adolescentes passam nesta rede social, percebemos um conjunto de resultados interessantes. Como se percebe na tabela seguinte, entre as várias relações significativas, destacam-se a relação positiva e forte entre o número de amigos na rede social e a intensidade de utilização $(r=.493, p<.01)$, e em particular entre a intensidade do uso do Facebook e o contacto com a rede offline $(r=.756, p<.01)$ e com a rede online $(r=.654, \mathrm{p}<.01)$, assim como entre a rede offline e online $(r=.712, p<.01)$. Percebe-se ainda a relação entre a autoestima e autorregulação $(r=.506$, $p<.01)$.

Com o recurso à regressão linear múltipla, com o método stepwise, conseguimos encontrar um modelo significativo $(F=102.770 . p<.01)$ e com um bom ajustamento $(R=.784)$, integrando quatro preditores da intensidade da utilização do Facebook. Destes, como se percebe na tabela 6 , o mais forte é a manutenção dos contactos offline - que explica $44.2 \%$ da variância -, seguido do número de amigos, tempo de utilização por dia e, por fim, a manutenção dos contactos online. 
Tabela 4.

Uso do Facebook, autoestima e autorregulação em função das classificações.

\begin{tabular}{lcc}
\hline & Classificação a Língua & Classificação a \\
& Portuguesa $(r s)$ & Matemática $(r s)$ \\
Uso do Facebook & .073 & .073 \\
Com rede offline & $-.127^{*}$ & .085 \\
Para contactos online & .087 & .078 \\
Autoestima & $.178^{* *}$ & $.173^{* *}$ \\
Autorregulação & -.017 & .032 \\
\hline
\end{tabular}

Nota. ** Correlação significativa para .01; * Correlação significativa para .05.

Tabela 5

Uso do Facebook, autoestima, autorregulação, número de amigos e tempo de utilização diário.

\begin{tabular}{|c|c|c|c|c|c|c|c|}
\hline & 1 & 2 & 3 & 4 & 5 & 6 & 7 \\
\hline 1. Uso do Facebook & 1 & & & & & & \\
\hline 2. Com rede offline & $.756^{* *}$ & 1 & & & & & \\
\hline 3. Para contactos online & $.654^{* *}$ & $.712^{* *}$ & 1 & & & & \\
\hline 4. Autoestima & $.194^{* *}$ & $.199 * *$ & $.146^{* *}$ & 1 & & & \\
\hline 5. Autorregulação & $.163^{* *}$ & $.180^{* *}$ & $.135^{* *}$ & $.506^{* *}$ & 1 & & \\
\hline 6. Número de amigos & $.493^{* *}$ & $.274^{* *}$ & $.282^{* *}$ & $.163^{* *}$ & .104 & 1 & \\
\hline 7. Tempo de uso diário & $.245^{* *}$ & -.007 & -.027 & .058 & .055 & .098 & 1 \\
\hline
\end{tabular}

Nota. ** Correlação significativa para 0.01 ; Correlação significativa para 0.05 .

Tabela 6

Preditores da intensidade da utilização do Facebook.

\begin{tabular}{|c|c|c|c|c|c|}
\hline Modelo & Preditores & $\mathrm{R}$ & $\mathrm{R}^{2}$ & $\mathrm{R}_{a d j}^{2}$ & $\Delta \mathrm{R}^{2}$ \\
\hline 1 & Comunicação com a rede offline & .665 & .442 & .440 & .442 \\
\hline 2 & Com rede offline; número de amigos & .738 & .545 & .542 & .103 \\
\hline 3 & $\begin{array}{l}\text { Com rede offline; número de amigos; } \\
\text { tempo por dia }\end{array}$ & .776 & .601 & .597 & .056 \\
\hline 4 & $\begin{array}{l}\text { Com rede offline; número de amigos; } \\
\text { tempo por dia; Com rede online. }\end{array}$ & .784 & .614 & .608 & .013 \\
\hline
\end{tabular}

Nota. $F=102.770 . \mathrm{p}<0.01$

Actualidades en Psicología, 31(123), 2017, 31-42 


\section{Discussão e Conclusão}

\section{Discussão e conclusões}

Nas últimas décadas, com a disseminação da internet e o acesso às novas tecnologias da informação e da comunicação, assistimos a alterações no relacionamento e na interação com os outros. A literatura, contudo, tem dados passos mais lentos na compreensão e na interpretação destes comportamentos, assim como dos seus significados junto de uma população que já nasceu na presença destas tecnologias. Nesse contexto, o presente estudo pretendia fazer uma leitura do uso das redes sociais, e em particular do Facebook, por adolescentes, identificando fatores que mais se associam ao seu uso.

Analisando os dados, parecem ir de encontro à literatura que diferencia aos significados do uso das redes sociais por género (Drabowicz, 2014; Kuss \& Griffiths, 2012; Subrahmanyam, Reich, Waechter \& Espinoza. 2008). Embora não sejam captadas diferenças na intensidade do uso, os rapazes parecem diferenciar-se pela maior utilização para manter os contactos offline, mas também para a procura de contactos online, no último caso com um tamanho de efeito já considerável. Já no que diz respeito à idade, a correlação positiva pode evidenciar algumas explicações na amostra ou culturais, uma vez que não seguem alguns estudos semelhantes realizados (Andreassen, Pallesen \& Griffiths, 2016; Junco, 2015; Kross et al., 2013; McAndrew \& Jeong, 2012; Pfeil, Arjan \& Zaphiris, 2009). Pelos dados, parece haver uma maior utilização da rede social pelos adolescentes mais velhos, na linha de outros estudos, em menor número, que sugere uma utilização maior entre os mais velhos (Calvani, Fini, Ranieri \& Picci, 2012; Helsper \& Eynon, 2009). Esses dados podem ainda ser influenciados pela idade mínima para o registo na rede social, pelas características desenvolvimentais da faixa etária, marcada por maior abertura à relação com os pares e tentativa de inclusão social ou pela capacidade económica das famílias. Isso pode ser contrastado com o previsível aumento da autoestima ao longo da escolaridade (Orth, Trzesniewski \& Robins, 2010) e estabilidade da autorregulação (Moilanen, Shaw
\& Fitzpatrick, 2010). Embora não existam muitas referências sobre a relação entre a escolaridade dos pais e o uso das redes sociais online, os resultados seguem uma tendência na literatura que associa a menor escolaridade da mãe a comportamentos de maior risco e menor autorregulação (Moilanen, Shaw \& Fitzpatrick, 2010).

Os resultados reforçam ainda a relação entre o uso das redes sociais e o desempenho escolar (Drabowicz, 2014; Junco, 2011, 2015). Embora a relação seja fraca, o tempo dedicado às redes sociais pode prejudicar o envolvimento em outras atividades, como as escolares, refletindo-se num menor desempenho. Isso reflete-se ainda pelo número de amigos da rede social. Embora não tenhamos diferenciado entre os amigos apenas online ou apenas offline, os resultados permitem perceber como as redes sociales online parecem ser determinantes para a intensidade do uso destas ferramentas (Khana, Wohn \& Ellison, 2014). A relação mais forte com os contactos offline pode pressupor alguma alienação das tarefas em que estão envolvidos, como referíamos antes com o desempenho escolar. No mesmo sentido, também a relação mais forte com a autoestima e os contactos offline (e.g. Gonzales \& Hancock, 2011; Vogel, Rose, Roberts \& Eckles, 2014; Wilcox \& Stephen, 2013), como no caso da autorregulação.

Das variáveis identificadas, o contacto com amigos offline aparece como o mais importante preditor do uso das redes sociais (Hew, 2011; Khana, Wohn \& Ellison, 2014). Apesar das potencialidades educativas e a diversidade de utilizações, o Facebook e as redes sociais parecem constituir-se como uma ferramenta essencialmente de contacto e proximidade entre pares, para partilhar informação e experiências maioritariamente entre pares que têm já uma relação de proximidade (e.g. Eklund, 2015; Hew, 2011). Estes dados podem fazer supor que, fruto da disseminação destas ferramentas, ao mesmo tempo que as mudanças sociais e educacionais limitam o tempo livre aos mais jovens, fazem destes artefactos recursos importantes para a sociabilização dos jovens. E dessa forma, pode fazer sentido a ausência de relação entre o uso das 
redes sociais, a autoestima e autorregulação apontada em alguns estudos. No entanto, são aspetos a ter em conta em futuros estudos, eventualmente recorrendo a outras metodologias para uma análise mais profunda e contextualizada. Na literatura encontramos dados muito distintos, que sugerem o benefício da sua utilização por adolescentes com baixa autoestima (Ellison, Steinfield \& Lampe, 2007; Steinfield, Ellison \& Lampe, 2008), outros estudos mostram uma relação negativa do uso e da importância do Facebook e a autoestima (Andreassen, Pallesen \& Griffiths, 2016; Blachio, Przepiorka \& Pantic, 2016). Não se encontrando estudos centrados na autorregulação e uso das redes sociais, podem estas variáveis não estar associadas diretamente mas mediadas por outras. Eventualmente, na linha de Rae e Lonborg (2015), a inclusão de medidas de motivações para a utilização destas redes sociais pode ser útil para uma visão mais completa do papel destas variáveis psicossociais. Enquanto campo de estudo emergente, futuras investigações devem continuar a ajudar-nos a perceber como estas novas gerações, ditas nativas digitais, percebem o uso destas ferramentas e as suas consequências a médio e longo prazo.

Apesar das conclusões, algumas limitações do estudo devem ser consideradas, como a amostra, recolhida por motivos de conveniência, no norte do país. Ainda, os dados recolhidos com recurso a instrumentos de autorrelato, permitem alguma desejabilidade social que deveriam ser esclarecidas, em futuros estudos, com amostras mais diversificadas e representativas da população, triangulando os resultados com recurso a dados qualitativos que permitam maior aprofundamento da informação.

\section{Referências}

Andreassen, C. S., Pallesen, S., \& Griffiths, M. D. (2017). The relationship between addictive use of social media, narcissism, and self-esteem: Findings from a large national survey. Addictive Behaviors, 64, 287-293. doi: 10.1016/j.addbeh.2016.03.006
Baym, N. K. (2010). Personal Connections in the Digital Age. Malden, MA: Polity Press.

Bennett, S. J., Maton, K. A., \& Kervin, L. K. (2008). The 'digital natives' debate: a critical review of the evidence. British Journal of EducationalTechnology, 39(5), 775-786. doi: 10.1111/j.1467-8535.2007.00793.x

Berners-Lee, T., Cailliau, R., Loutonen, A., Nielsen, H. F., \& Secret, A. (1994). The World-Wide Web. Communications of the ACM, 37(8), 76-82.

Blachio, A., Przepiorka, A., \& Pantic, I. (2016). Association between Facebook addiction, selfesteem and life satisfaction: A cross-sectional study. Computers in Human Behavior, 55, 701-705. doi: 10.1016/j.chb.2015.10.026

Boulos, M. N., \& Weeler, S. (2007). The emerging Web 2.0 social software: an enabling suite of sociable technologies in health and health care education. Health Information and Libraries Journal, 24(1), 2-23. doi: 10.1111/j.1471-1842.2007.00701.x

Boyd, D. M. (2008). Why youth (heart) social network sites: The role of networked publics in teenage social life. In D. Buckingham (Ed.), Youth, Identity, and Digital Media (pp. 119-142). Cambridge, MA: MIT Press.

Boyd, D. M., \& Ellison, N. B. (2007). Social network sites: Definition, history, and scholarship. Journal of Computer-Mediated Communication, 13(1), 210-230. doi: 10.1111/j.1083-6101.2007.00393.x

Buckley, C., Hastings, A., \& Mottershead, R. (2009). Using Web 2.0 Technologies to Build Learning Communities. Paper presented to the International Association for Development of the Information Society (IADIS), Barcelona.

Calvani, A., Fini, A., Ranieri, M., \& Picci, P. (2012). Are young generations in secondary school digitally competent? A study on Italian teenagers. Computers \& Education, 58(2), 797-807. doi: 10.1016/j. compedu.2011.10.004 
Campbell, D. E. (2013). Social networks and political participation. Annual Review of Political Science, 16, 3348. doi: 10.1146/annurev-polisci-033011-201728

Capua, I. D. (2012). A literature review of research on Facebook use. The Open Communication Journal, 6, 3742. doi: 10.2174/1874916X01206010037

Carvalho, A. A. (2008). Manual de Ferramentas da Web 2.0 para Professores. Lisboa: Direcção-Geral de Inovação e de Desenvolvimento Curricular do Ministério da Educação.

Castells, M. (2009). Communication Power. Oxford: Oxford University Press.

Charnigo, L., \& Barnett-Ellis, P. (2007). Checking out Facebook.com: The impact of a digital trend on academic libraries. Information Technology and Libraries, 26(1), 23-34. doi: 10.6017/ital.v26i1.3286

Cohen, J. (1992). A Power Primer. Psychological Bulletin, 112(1), 155-159. doi: 10.1037/0033-2909.112.1.155

Cornu, B. (2011). Digital natives: How do they learn? How to teach them? Moscow: UNESCO.

Deans, P. C. (2008). Social Software and Web 2.0 Technology Trends. New York: Information Science Reference.

Dias, P. C., Garcia del Castillo, J. A., \& Schwarzer, R. (2008). Estudo de adaptação da Escala de AutoRegulação (Schwarzer, 1999) para a população portuguesa. Em A. P. Noronha, C. Machado, L. Almeida, M. Gonçalves, S. Martins, \& V. Ramalho (Coords.), Actas da XIII Conferência Internacional de Avaliação Psicológica: Formas e contextos [edição em cdrom] (s.p.). Braga, Portugal: Psiquilibrios Edições.

Dias, P. C., Garcia del Castillo, J. A., Castillo-López, A. G., \& Sorte, A. (2015). Facebook use and adolescents selfesteem: Contributes of the adaptation of Ellison Facebook use scales. Poster apresentado no III Congreso Internacional de Ciencias de la Educación y del Desarrollo. San Sebastian, Espanha.

Drabowicz, T. (2014). Gender and digital usage inequality among adolescents: A comparative study of 39 countries. Computers \& Education, 74, 98-111. doi: 10.1016/i.compedu.2014.01.016
Eklund, L. (2015). Bridging the online/offline divide: The example of digital gaming. Computers in Human Behavior, 53, 527-535. doi: 10.1016/j. chb.2014.06.018

Ell, K. (1984). Social networks, social support, and health status: A Review. Social Service Review, 58(1), 133-149.

Ellison, N. B., Steinfield, C., \& Lampe, C. (2007). The benefits of Facebook "friends": Social capital and college students' use of online social network sites. Journal of Computer-Mediated Communication, 12(4), 1143-1168. doi: 10.1111/j.1083-6101.2007.00367.x

Faber, A. D., \& Wasserman, S. (2002). Social support and social networks: synthesis and review. In J. A. Levy, B. A. Pescosolido (ed.), Social Networks and Health: Advances in Medical Sociology (Vol. 8, pp. 29 72). New York Emerald Group Publishing.

Garcia del Castillo, J. A., Dias, P. C., \& Perim, P. C. (2012). Auto-regulação e consumo de substâncias na adolescência. Evidencias de um estudo exploratório. Psicologia: Reflexão e Crítica, 25(2), 238247. doi: 10.1590/S0102-79722012000200005

Gardner, T., Dishion, T., \& Connell, A. (2008). Adolescent self-regulation as resilience: Resistance to antisocial behavior within the deviant peer context. Journal of Abnormal Child Psychology, 36(2), 273-284. doi: 10.1007/s10802-007-9176-6

Gonzales, A. L., \& Hancock, J. T. (2011). Mirror, mirror on my Facebook wall: effects of exposure to Facebook on self-esteem. Cyberpsychology, Behavior, \& Social, 14, 79-83. doi: 10.1089/cyber.2009.0411

Grieve, R., Indiain, M., Witteveen, K., Tolan, G., \& Marrington, J. (2013). Face-to-face or Facebook: Can social connectedness be derived online? Computers in Human Behavior, 29(3), 604-609. doi: 10.1016/i.chb.2012.11.017

Helsper, E., \& Eynon, R. (2009). Digital natives: Where is the evidence? British Educational Research Journal, 36(3), 1-18. doi: 10.1080/01411920902989227 
Hendrix, D., Chiarella, D., Hasman, L., Murphy, S., \& Zafron, M. L. (2009). Use of Facebook in academic health sciences libraries. Journal of the Medical Library Association, 97(1), 44-47. doi: 10.3163/15365050.97.1.008

Hew, K. F. (2011). Students' and teachers' use of Facebook. Computers in Human Behavior, 27, 662676. doi:10.1016/i.chb.2010.11.020

Jeon, K. C., \& Goodson, P. (2015). US adolescents' friendship networks and health risk behaviors: a systematic review of studies using social network analysis and Add Health data. PeerJ, 3, e1052; doi: $\underline{10.7717 / \text { peeri. }} .1052$

Johnston, K., Tanner, M., Lalla, N., \& Kawalski, D. (2013). Social capital: the benefit of Facebook 'friends'. Behaviour \& Information Technology, 32(1), 2436. doi: 10.1080/0144929X.2010.550063

Junco, R. (2011). The relationship between frequency of Facebook use, participation in Facebook activities, and student engagement. Computers \& Education, 58, 162-171. doi: 10.1016/i.compedu.2011.08.004

Junco, R. (2015). Student class standing, Facebook use, and academic performance. Journal of Applied Developmental Psychology, 36, 18-29. doi: 10.1016/j. appdev.2014.11.001

Kalpidou, M., Coustin, D., \& Morris, J. (2011). The relationship between Facebook and the well-being of undergraduate college students. Cyberpsychology, Behavior and Social Networking, 14(4), 183-189. doi: 10.1089/cyber.2010.0061

Khana, M. L.,Wohn, D. Y., \& Ellison, N. B. (2014). Actual friends matter: An internet skills perspective on teens' informal academic collaboration on Facebook. Computers \& Education, 79, 138-147. doi: 10.1016/i.compedu.2014.08.001

Kross, E., Verduyn, P., Demiralp, E., Park, J., Lee, D. S., Lin, N., ... Ybarra, O. (2013). Facebook Use Predicts Declines in Subjective Well-Being in Young Adults. PLoS ONE, 8(8), e69841. doi:10.1371/ journal.pone.0069841
Kuss, D. J., \& Griffiths, M. D. (2012). Internet gaming addiction: A systematic review of empirical research. International Journal of Mental Health and Addiction, 10, 278. doi: 10.1007/s11469-011-9318-5

Lampe, C., Ellison, N., \& Steinfield, C. (2007). A familiar Face(book): Profile elements as signals in an online social network. Proceedings of the SIGCHI Conference on Human Factors in Computing Systems (pp. 435-444). New York: ACM Press.

Martire, L. M., \& Franks, M. M. (2014). The role of social networks in adult health: Introduction to the special issue. Health Psychology, 33(6), 501-504. doi:10.1037/hea 0000103

McAndrew, F. T., \& Jeong, H. S. (2012). Who does what on Facebook? Age, sex, and relationship status as predictors of Facebook use. Computers in Human Behavior, 28, 2359-2365. doi: 10.1016/j. chb.2012.07.007

Mitchell, J. C. (1974). Social Networks. Annual Review of Anthropology, 3, 279-299. doi:10.1146/annurev. an.03.100174.001431

Moilanen, K. L., Shaw, D. S., \& Fitzpatrick, A. (2010). Self-regulation in early adolescence: Relations with mother-son relationship quality and maternal regulatory support and antagonism. Journal of Youth and Adolescence, 39(11), 1357-1367. doi: 10.1007/ s10964-009-9485-x

O'Reilly, T. (2006). Web 2.0 Compact Definition: Trying Again. Recuperado de http://radar.oreilly. com/2006/12/web-20-compact-definition-tryi.html

Orth, U., Trzesniewski, K. H., \& Robins, R. W. (2010). Self-esteem development from young adulthood to old age: A cohort-sequential longitudinal study. Journal of Personality and Social Psychology, 98(4), 645658. doi: 10.1037/a0018769

Pfeil, U., Arjan, R., \& Zaphiris, P. (2009). Age differences in online social networking - A study of user profiles and the social capital divide among teenagers and older users in MySpace. Computers in Human Behavior, 25, 643-654. doi: 10.1016/i. chb.2008.08.015 
Pontes, H. M., Andreassen, C. S., \& Griffiths, M. D. (2016). Portuguese Validation of the Bergen Facebook Addiction Scale: An empirical study. International Journal of Mental Health and Addiction, 14(6), 1062-1073. doi:10.1007/s11469-016-9694-y

Pontes, H. M., Patrão, I. M., \& Griffiths, M. D. (2014). Portuguese validation of the Internet Addiction Test: An empirical study. Journal of Behavioral Addictions, 3(2), 107-114. doi: 10.1556/JBA.3.2014.2.4

Prensky, M (2001). Digital natives, digital immigrants: Part 1. On the Horizon, 9(5), 1 - 6.

Rico-Uribe, L. A., Caballero, F. F., Olaya, B., TobiaszAdamczyk, B., Koskinen, S., Leonardi, M, ... Miret, M. (2016). Loneliness, social networks, and health: A cross-sectional study in three countries. PLOS ONE 11(1), e0145264. doi: 10.1371/journal. pone. 0145264

Romano, A., Negreiros, J., \& Martins, T. (2007). Contributos para a validação da escala de autoestima de Rosenberg numa amostra de adolescentes da região interior norte do país. Psicologia, Saúde \& Doenças, 8(1), 107-114.

Rosenberg, M. (1965). Society and the adolescent self-image. Princeton: Princeton University Press.

Schwarzer, R. (1999). Self-Regulation Scale. Recuperado de http://userpage.fu-berlin.de/ health/selfreg g.htm

Smith, K. P., \& Christakis, N. A. (2008). Social networks and health. Annual Review of Sociology, 34, 405-429. doi: 10.1146/annurev.soc.34.040507.134601

Steinfield, C., Ellison, N. B., \& Lampe, C. (2008). Social capital, self-esteem, and use of online social network sites: A longitudinal analysis. Journal of Applied Developmental Psychology, 29(6), 434-445. doi:10.1016/j.appdev.2008.07.002

Subrahmanyam, K., Reich, S. M., Waechter, N., \& Espinoza, G. (2008). Online and offline social networks: Use of social networking sites by emerging adults. Journal of Applied Developmental Psychology, 29, 420-433. doi: 10.1016/j.appdev.2008.07.003

Tapscott, D. (1998). Growing up digital: The rise of the net generation. New York: McGraw-Hill.

Tsai, A.C., \& Papachristos, A.V. (2015). From social networks to health: Durkheim after the turn of the millennium. Social Science \& Medicine, 125, 1-7. doi: 10.1016/j.socscimed.2014.10.045

Umberson, D., Crosnoe, R., \& Reczek, C. (2010). Social relationships and health behavior across the life course. Annual Review of Sociology, 36, 139-157. doi: 10.1146/annurev-soc-070308-120011

Uribe-Tirado, A., \& Echavarría-Ramírez, A-F. (2008). Facebook como red de profesionales de bibliotecología, documentación y archivística en Iberoamérica. El Profesional de la Información, 17(6), 670-676.

Valkenburg, P. M., \& Peter, J. (2009). Social consequences of the internet for adolescents: A decade of research. Current Directions in Psychological Science, 18(1), 1-5. doi: 10.1111/j.14678721.2009.01595.x

Videras, J. (2013). Social networks and the environment. Annual Review of Resource Economics, 5, 211-226. doi: 10.1146/annurev-resource-091912-151912

Vogel, E. A., Rose, J. P., Roberts, L. R., \& Eckles, K. (2014). Social comparison, social media, and selfesteem. Psychology of Popular Media Culture, 3(4), 206222. doi: $10.1037 / \mathrm{ppm} 0000047$

Wilcox, K., \& Stephen, A. T. (2013). Are close friends the enemy? Online social networks, self-esteem, and self-control. Journal of Consumer Research, 40, 90-103. doi: $10.1086 / 668794$ 